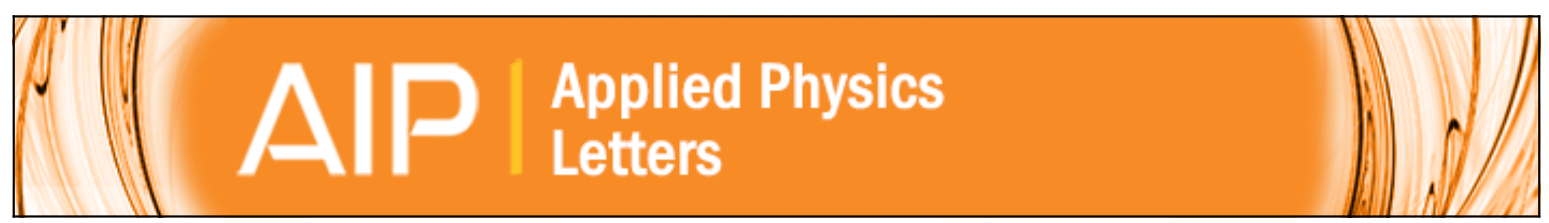

\title{
Nanoscale gadolinium oxide capping layers on compositionally variant gate dielectrics
}

H. N. Alshareef, J. A. Caraveo-Frescas, and D. K. Cha

Citation: Applied Physics Letters 97, 202108 (2010); doi: 10.1063/1.3519363

View online: $h t t p: / / d x . d o i . o r g / 10.1063 / 1.3519363$

View Table of Contents: http://scitation.aip.org/content/aip/journal/apl/97/20?ver=pdfcov

Published by the AIP Publishing

\section{Articles you may be interested in}

Experimental and theoretical investigation of the effect of $\mathrm{SiO} 2$ content in gate dielectrics on work function shift induced by nanoscale capping layers

Appl. Phys. Lett. 101, 112902 (2012); 10.1063/1.4747805

Anomalous positive flatband voltage shifts in metal gate stacks containing rare-earth oxide capping layers

Appl. Phys. Lett. 100, 102111 (2012); 10.1063/1.3692580

Effects of a Gd capping layer on electrical characteristics of metal-oxide-semiconductor field effect transistors with a TaC gate electrode and a HfSiON gate dielectric

Appl. Phys. Lett. 95, 192113 (2009); 10.1063/1.3264086

Band offsets of high $\mathrm{K}$ gate oxides on III-V semiconductors

J. Appl. Phys. 100, 014111 (2006); 10.1063/1.2213170

Ti gate compatible with atomic-layer-deposited $\mathrm{HfO} 2$ for $\mathrm{n}$-type metal-oxide-semiconductor devices Appl. Phys. Lett. 86, 092107 (2005); 10.1063/1.1871362

Want to publish your paper in the \#1 MOST CITED journal in applied physics?

With Applied Physics Letters, you can.

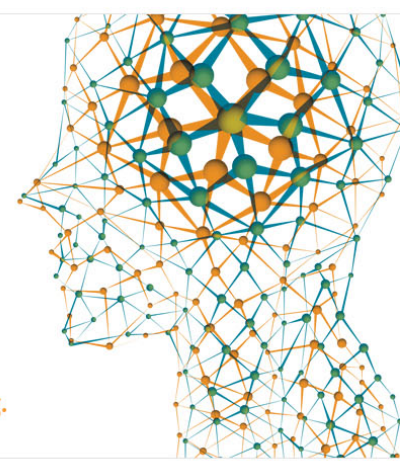




\title{
Nanoscale gadolinium oxide capping layers on compositionally variant gate dielectrics
}

\author{
H. N. Alshareef, ${ }^{1, a)}$ J. A. Caraveo-Frescas, ${ }^{1}$ and D. K. Cha ${ }^{2}$ \\ ${ }^{1}$ Materials Science and Engineering, King Abdullah University of Science and Technology, \\ Thuwal 23955-6900, Saudi Arabia \\ ${ }^{2}$ Advanced Nanofabrication, Imaging and Characterization Laboratory, King Abdullah University of Science \\ and Technology, Thuwal 23955-6900, Saudi Arabia
}

(Received 5 September 2010; accepted 28 October 2010; published online 19 November 2010)

\begin{abstract}
Metal gate work function enhancement using nanoscale $(1.0 \mathrm{~nm}) \mathrm{Gd}_{2} \mathrm{O}_{3}$ interfacial layers has been evaluated as a function of silicon oxide content in the $\mathrm{Hf}_{x} \mathrm{Si}_{y} \mathrm{O}_{z}$ gate dielectric and process thermal budget. It is found that the effective work function tuning by the $\mathrm{Gd}_{2} \mathrm{O}_{3}$ capping layer varied by nearly $400 \mathrm{mV}$ as the composition of the underlying dielectric changed from $0 \%$ to $100 \% \mathrm{SiO}_{2}$, and by nearly $300 \mathrm{mV}$ as the maximum process temperature increased from ambient to $1000{ }^{\circ} \mathrm{C}$. A qualitative model is proposed to explain these results, expanding the existing models for the lanthanide capping layer effect. (C) 2010 American Institute of Physics. [doi:10.1063/1.3519363]
\end{abstract}

Metal gate work function engineering in transistor devices is a subject of great interest in the semiconductor community. Rare-earth oxide capping has been demonstrated as an effective method to tune the work function of metal gates on Hf-based dielectrics. ${ }^{1} \mathrm{La}_{2} \mathrm{O}_{3}$ has received the greatest attention, although $\mathrm{Gd}_{2} \mathrm{O}_{3}$ is especially significant because of its potential in non-Si based devices (although normally in solid solution with $\left.\mathrm{Ga}_{2} \mathrm{O}_{3}\right){ }^{2,3}$ Most analysis of the role of the lanthanides focused on the thickness and composition of the capping rare-earth oxide layer. However, the dependence on the underlying gate dielectric composition and thermal budget has not been systematically investigated. In this article, we show that the amount of work function tuning by the $\mathrm{Gd}_{2} \mathrm{O}_{3}$ capping layer strongly depends on the $\mathrm{SiO}_{2}$ content in the underlying $\mathrm{Hf}_{x} \mathrm{Si}_{y} \mathrm{O}_{z}$ gate dielectric and propose a mechanism to explain the observed results.

Capacitors were built on heavily doped p-type substrates with a dopant concentration of $1.2 \times 10^{18} \mathrm{~cm}^{-3}$. Pregate cleaning was performed using diluted hydrofluoric acid (HF) followed by $\mathrm{O}_{3}$-last cleaning. The $\mathrm{SiO}_{2}$ gate dielectric was grown using thermal oxidation, while the $\mathrm{HfO}_{2}$ and $\mathrm{Hf}_{x} \mathrm{Si}_{y} \mathrm{O}_{z}$ films $(2.0 \mathrm{~nm})$ were grown by atomic layer deposition. The $1.0 \mathrm{~nm} \mathrm{Gd}{ }_{2} \mathrm{O}_{3}$ interfacial layer and $10 \mathrm{~nm}$ TaN gate electrode were then deposited at room temperature using a physical vapor deposition system. The stack was then capped with polycrystalline silicon (poly-Si), implanted by phosphorous, and then annealed at $1000{ }^{\circ} \mathrm{C}$ for $10 \mathrm{~s}$ to activate the dopants. In some samples, no poly-Si was deposited, instead, a $40 \mathrm{~nm}$ thick TaN was used to allow studying of the stack at lower temperatures (no poly-Si activation). The backside of the wafer was coated with $\mathrm{Al}$ metal to improve the electrical contact. The $\mathrm{C}-\mathrm{V}$ curves were fitted using the NCSU model to extract the equivalent oxide thickness (EOT), flat-band voltage $\left(V_{\mathrm{FB}}\right)$, and substrate doping concentration. ${ }^{4}$ The effective work function of each electrode was extracted from the plots of $V_{\mathrm{FB}}$ versus EOT using a series of $\mathrm{SiO}_{2}$ thicknesses $(1.0-4.0 \mathrm{~nm})$ formed by wet-etching of a thermally grown $4.0 \mathrm{~nm} \mathrm{SiO}_{2}$ layer.

\footnotetext{
a) Author to whom correspondence should be addressed. Electronic mail: husam.alshareef@kaust.edu.sa.
}

Figure 1 shows the capacitance-voltage $(\mathrm{C}-\mathrm{V})$ curves obtained from TaN/ $\mathrm{Gd}_{2} \mathrm{O}_{3} / \mathrm{Hf}_{x} \mathrm{Si}_{y} \mathrm{O}_{z} / \mathrm{SiO}_{2} / \mathrm{Si}$ stacks. Figure 1 demonstrates that these devices have well-behaved $\mathrm{C}-\mathrm{V}$ curves and show a marked shift in $V_{\mathrm{FB}}$ as the $\mathrm{SiO}_{2}$ content in the gate dielectric increases from $0 \%$ to $100 \%$ in $\mathrm{Hf}_{x} \mathrm{Si}_{y} \mathrm{O}_{z}$ $\left[\% \mathrm{SiO}_{2}=y /(x+y)^{*} 100\right]$. The dashed curve is for a reference device without $\mathrm{Gd}_{2} \mathrm{O}_{3}\left(\mathrm{TaN} / \mathrm{Hf}_{0.4} \mathrm{Si}_{0.6} \mathrm{O}_{z} / \mathrm{SiO}_{2} / \mathrm{Si}\right)$. With the $1.0 \mathrm{~nm} \mathrm{Gd} \mathrm{O}_{3}$ capping layer, nearly $400 \mathrm{mV}$ shift in $V_{\mathrm{FB}}$ is observed. In fact, the absolute value of $V_{\mathrm{FB}}$ shift $\left(\Delta V_{\mathrm{FB}}\right)$ varies nearly linearly with the fraction of $\mathrm{SiO}_{2}$ in the gate dielectric, $\Delta V_{\mathrm{FB}}(\mathrm{mV})=a X_{\mathrm{SiO} 2}+b$, where $X_{\mathrm{SiO} 2}=y /(x+y), a$ and $b$ are constants that depend on the stack and are equal to 370 and $60 \mathrm{mV}$, respectively, for the $\mathrm{Gd}_{2} \mathrm{O}_{3} / \mathrm{Hf}_{x} \mathrm{Si}_{y} \mathrm{O}_{z}$ system. Figure 2 shows $V_{\mathrm{FB}}$ versus EOT plots for four devices: (a) reference device without $\mathrm{Gd}_{2} \mathrm{O}_{3}$ and [(b)-(d)] devices with $1.0 \mathrm{~nm} \mathrm{Gd}_{2} \mathrm{O}_{3}$ capping layer deposited on $\mathrm{Hf}_{x} \mathrm{Si}_{y} \mathrm{O}_{z}$ dielectric containing $0 \%, 60 \%$, and $100 \% \mathrm{SiO}_{2}$, respectively. The curve shows a shift in $V_{\mathrm{FB}}$ as a function of $\% \mathrm{SiO}_{2}$ independent of the interfacial $\mathrm{SiO}_{2}$ layer thickness (which was

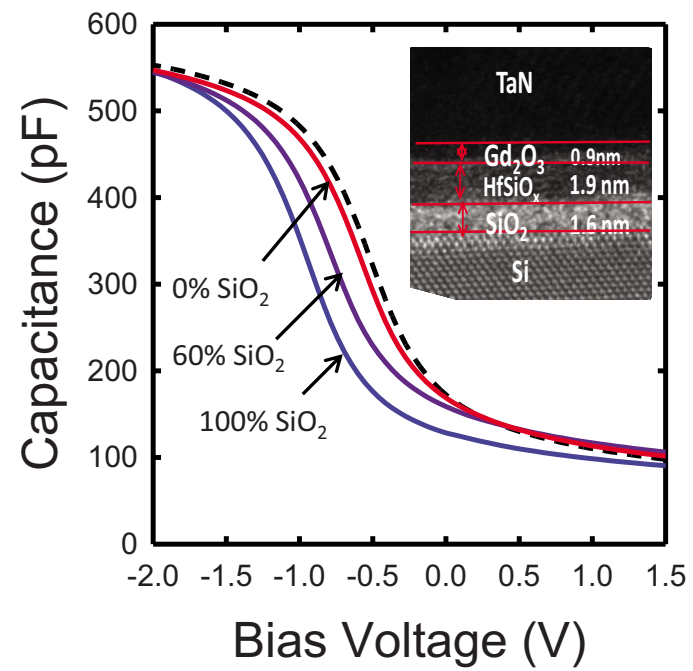

FIG. 1. (Color online) C-V curves of devices with nearly the same EOT showing the gradual shift in $\mathrm{C}-\mathrm{V}$ curve with $\% \mathrm{SiO}_{2}$ in the gate dielectric. The dotted line is for a device without the $\mathrm{Gd}_{2} \mathrm{O}_{3}$ capping layer. The inset shows a TEM X-section of the stack. 


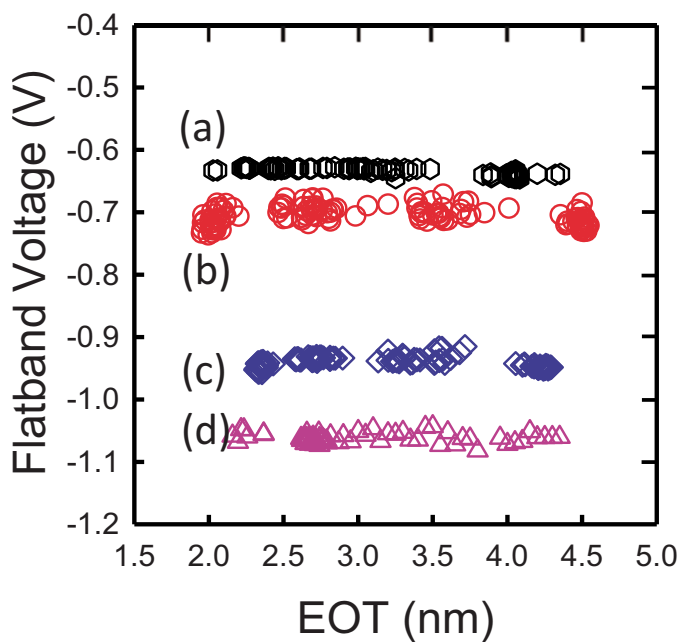

FIG. 2. (Color online) $V_{\mathrm{FB}}$-EOT plots showing the shift in $V_{\mathrm{FB}}$ over a range of EOTs for samples (a) without $\mathrm{Gd}_{2} \mathrm{O}_{3}$ capping layer and for samples with $1.0 \mathrm{~nm} \mathrm{Gd} \mathrm{O}_{3}$ capping layer where gate dielectric contains (b) $0 \% \mathrm{SiO}_{2}$, (c) $60 \% \mathrm{SiO}_{2}$, and (d) $100 \% \mathrm{SiO}_{2}$. Negligible interfacial fixed charges are obtained.

varied from 1.0 to $4.0 \mathrm{~nm}$, giving $2.0-4.5$ EOTs). Furthermore, it is interesting to note that the fixed-charge concentration in all these devices is relatively small, around 1-6 $\times 10^{10} \mathrm{~cm}^{-2}$, as calculated from the slope of the $V_{\mathrm{FB}}$ versus EOT plots in Fig. 2. The effective work function of the TaN metal gate used here was extracted from Fig. 2 and is shown in Fig. 3 for stacks with $1.0 \mathrm{~nm} \mathrm{Gd}_{2} \mathrm{O}_{3}$ on $\mathrm{HfO}_{2}\left(0 \% \mathrm{SiO}_{2}\right)$, $\mathrm{Hf}_{0.4} \mathrm{Si}_{0.6} \mathrm{O}_{z}\left(60 \% \mathrm{SiO}_{2}\right)$, and $\mathrm{SiO}_{2}\left(100 \% \mathrm{SiO}_{2}\right)$. Note that the largest shift in the work function caused by the $\mathrm{Gd}_{2} \mathrm{O}_{3}$ capping layer occurs for the $100 \% \mathrm{SiO}_{2}$ dielectric, consistent with the $V_{\mathrm{FB}}$ shifts observed earlier.

It has been shown that rare-earth oxides deposited directly on $\mathrm{SiO}_{2}$ tend to diffuse toward the $\mathrm{SiO}_{2} / \mathrm{Si}$ layer often resulting in silicate formation even at temperatures as low as $400{ }^{\circ} \mathrm{C} .{ }^{5-8}$ It is not surprising that such reactions occur since the free energy of oxide formation $\Delta \mathrm{G}_{\mathrm{f}}$ for rare-earth oxides is significantly more negative than $\mathrm{SiO}_{2}(-856$ versus $-1730 \mathrm{~kJ} / \mathrm{mol}$ for $\mathrm{SiO}_{2}$ and $\mathrm{Gd}_{2} \mathrm{O}_{3}$, respectively). ${ }^{9}$ In our samples, Gd intensity profiles were obtained from electron energy loss spectroscopy (EELS) analysis and show that Gd has clearly diffused within the gate stack, although the origi-

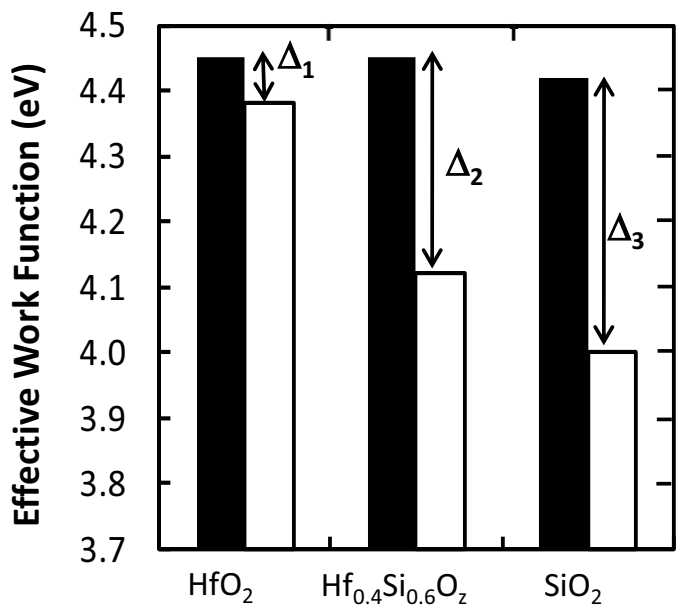

FIG. 3. Extracted effective work function (EWF) values as a function of $\% \mathrm{SiO}_{2}$ in the gate dielectric where all devices had $1.0 \mathrm{~nm} \mathrm{Gd}_{2} \mathrm{O}_{3}$ capping layer. The magnitude of the increase in EWF is marked by $\Lambda_{1}, \Delta_{2}$, and $\Delta_{3}$.

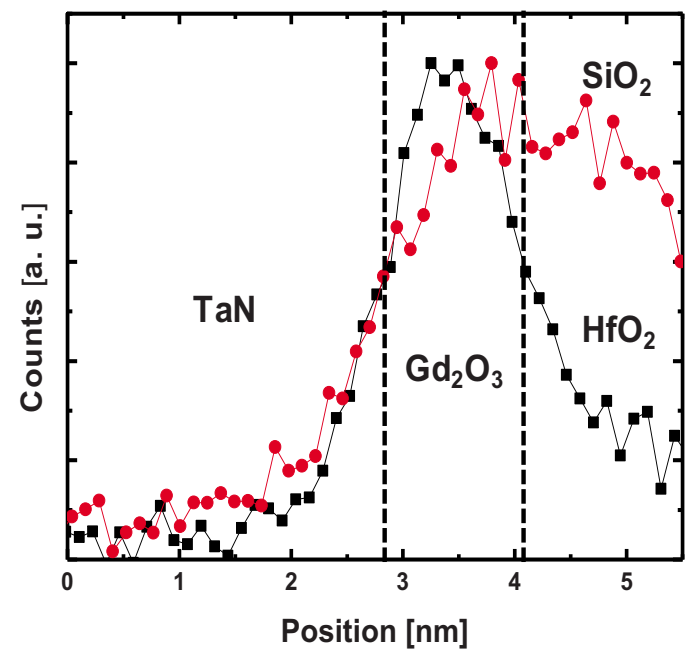

FIG. 4. (Color online) EELS chemical profile recorded across $\mathrm{Gd}_{2} \mathrm{O}_{3}$ containing gate stacks. The results show the $\mathrm{Gd}$ element signal variation with depth in $\mathrm{Gd}_{2} \mathrm{O}_{3} / \mathrm{SiO}_{2}$ and $\mathrm{Gd}_{2} \mathrm{O}_{3} / \mathrm{HfO}_{2}$ stacks. The $\mathrm{Gd}$ has diffused toward the $\mathrm{Si}$ interface, but not into TaN. Furthermore, the normalized concentration plot shows that more Gd has diffused in the case of $\mathrm{SiO}_{2}$ than $\mathrm{HfO}_{2}$

nal $\mathrm{Gd}_{2} \mathrm{O}_{3}$ capping layer remains distinct (Fig. 4). Figure 4 shows that the Gd levels are significantly higher in the gate dielectric region compared to the TaN gate region, indicating downward diffusion. Furthermore, Fig. 4 shows that the normalized intensities of $\mathrm{Gd}$ are higher in the case of underlying $\mathrm{SiO}_{2}$ dielectric than in the case of $\mathrm{HfO}_{2}$ dielectric, providing significant information to help understand the observed device behaviors, as will be discussed shortly. The $V_{\mathrm{FB}}$ shift observed with lanthanide capping on Hf-based dielectrics has been correlated with three main mechanisms including (1) positive fixed charges generated due to substitution of lanthanide cations on $\mathrm{Hf}^{4+}$ sites forming positively charged $\mathrm{O}$ vacancies,$^{10}$ (2) dipole formation involving the rare-earth (RE) atoms at the interface, ${ }^{11,12}$ and (3) work function of the metal of the metal-oxide capping layer $(\mathrm{Gd}, \mathrm{La}$, etc.) and dielectric constant discontinuity. ${ }^{13}$ Relating these models to $\% \mathrm{SiO}_{2}$ content in the gate dielectric or thermal budget has not been specifically discussed.

The low values of fixed charges in our stacks for all dielectric compositions studied $\left(1-5 \times 10^{10} \mathrm{~cm}^{-2}\right)$ clearly show that the observed $V_{\mathrm{FB}}$ shifts cannot simply be due to fixed charge accumulation (such as oxygen vacancies generated from $\mathrm{Gd}^{3+}$ substitution on $\mathrm{Hf}^{4+}$ or $\mathrm{Si}^{4+}$ sites). This assertion is also supported by the fact that $\mathrm{Al}$ and La capping layers produce shifts in opposite directions despite having the same valence, ruling out the fixed charge model. ${ }^{14,15}$ On the other hand, the dipole model can predict the $V_{\mathrm{FB}}$ shifts both in sign and in magnitude by calculating the dipole moment of the $\mathrm{RE}-\mathrm{O}, \mathrm{Si}-\mathrm{O}, \mathrm{Hf}-\mathrm{O}$ bonds using bond distances and electronegativity. ${ }^{11}$ To determine if the dipole model can explain the composition and temperature dependence of the $V_{\mathrm{FB}}$ shifts caused by $\mathrm{Gd}_{2} \mathrm{O}_{3}$ in our samples, we reiterate that our data indicate that significant $\mathrm{Gd}$ diffusion takes place in our gate stacks. The driving force for this diffusion is most likely the difference in the free energy of oxide formation between the lanthanide capping layer and $\mathrm{SiO}_{2}$ as mentioned earlier. Due to the presence of the $\mathrm{SiO}_{2}$ interfacial layer, there will always be a higher concentration of $\mathrm{SiO}_{2}$ near silicon substrate which acts to drive the lanthanide element layer closer to the bottom interface as previously 


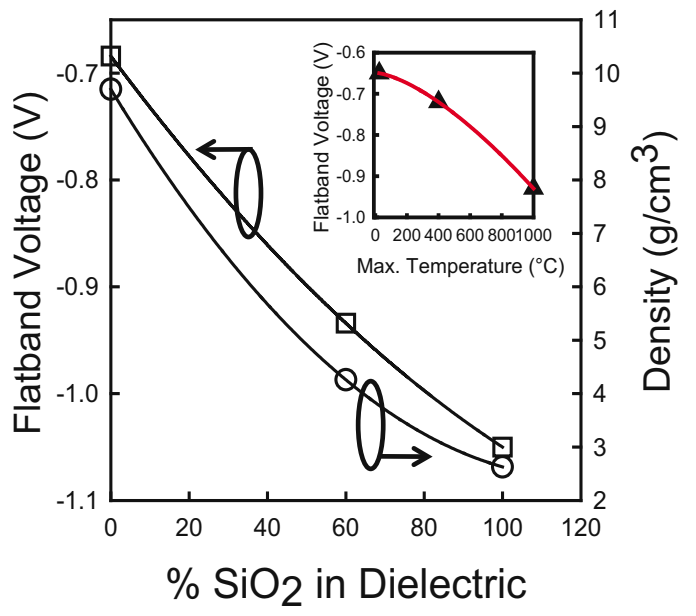

FIG. 5. (Color online) Results showing the $V_{\mathrm{FB}}$ shift as a function of $\% \mathrm{SiO}_{2}$ in the gate dielectric and gate dielectric density. The inset shows the impact of maximum thermal budget on $V_{\mathrm{FB}}$ shift for $60 \% \mathrm{SiO}_{2}$ dielectric.

demonstrated. ${ }^{4,6,7}$ The kinetics (rate) of this diffusion reaction depends on many factors. In our data, Fig. 5 shows two important effects. One is that the atomic density of the gate dielectric correlates well with the observed $V_{\mathrm{FB}}$ shifts. $\mathrm{HfO}_{2}$ has a relatively large density of $9.7 \mathrm{~g} / \mathrm{cm}^{3}$, hence producing the smallest $V_{\mathrm{FB}}$ shifts. This is believed to be a result of limited lanthanide metal diffusion through the dense $\mathrm{HfO}_{2}$ to the lower interface and possibly leading to smaller dipole concentration. In an open structure like that of $\mathrm{SiO}_{2}$ (tetrahedral network), the lanthanide atoms can more easily reach the interface. The $\mathrm{Hf}_{0.4} \mathrm{Si}_{0.6} \mathrm{O}_{z}$ dielectric has an intermediate density between $\mathrm{SiO}_{2}$ and $\mathrm{HfO}_{2}$, resulting in intermediate $V_{\mathrm{FB}}$ shift. The inset of Fig. 5 also shows that the maximum temperature reached by the device causes a big $V_{\mathrm{FB}}$ shift. In fact, the absolute value of the shift follows an Arrhenius behavior indicating a thermally activated process, most likely diffusion of the lanthanide atoms. Quantitatively, the absolute value of $V_{\mathrm{FB}}$ shift relative to samples without $\mathrm{Gd}_{2} \mathrm{O}_{3}$ is given by $\Delta V_{\mathrm{FB}}=a \exp \left(-Q^{*} 10^{3} / k T\right)$, with $a$ equal to $1005 \mathrm{mV}$ and $Q=0.13 \mathrm{eV}$, a relatively small activation energy. The evidence for this thermally activated process could also be seen in the work of Kirsch et al. ${ }^{11}$ where they proposed the dipole model. They found that $\mathrm{Sr}$ produced the largest amount of $V_{\mathrm{FB}}$ shift while Sc produced the smallest amount of the $V_{\mathrm{FB}}$ shift. ${ }^{11}$ While this difference was largely attributed to electronegativity differences, it is interesting to note that the melting point of $\mathrm{Sr}$ is $795{ }^{\circ} \mathrm{C}$ while that of $\mathrm{Sc}$ is $1539{ }^{\circ} \mathrm{C}$, which in our judgment was a key reason for the large difference in $V_{\mathrm{FB}}$ shift reported, as more significant diffusion and accumulation of $\mathrm{Sr}$ occurred at the lower interface. This likely resulted in higher dipole concentration at the interface in the case of Sr.

The model of Lin et al. ${ }^{13}$ suggests that the $V_{\mathrm{FB}}$ shift relates to the work function of the metal in the metal-oxide capping layer and predicts that capping layers which produce large discontinuity in the dielectric constant will produce the maximum $V_{\mathrm{FB}}$ shifts. Since the dielectric constant difference $\left(\Delta \varepsilon_{\mathrm{r}}\right)$ between $\mathrm{Gd}_{2} \mathrm{O}_{3}$ (capping layer in our samples) and the underlying gate dielectric increases with $\% \mathrm{SiO}_{2}$ in the gate dielectric, the model seems to be consistent with the observation that the $V_{\mathrm{FB}}$ shifts increase with $\% \mathrm{SiO}_{2}$ in gate dielectric. Assuming a dielectric constant of 22, 25, 14, and 4 for $\mathrm{Gd}_{2} \mathrm{O}_{3}, \mathrm{HfO}_{2}, \mathrm{Hf}_{0.4} \mathrm{Si}_{0.6} \mathrm{O}_{z}$, and $\mathrm{SiO}_{2}$, respectively, we calculate that the measured $V_{\mathrm{FB}}$ shifts are linearly related to $\Delta \varepsilon_{\mathrm{r}}$, where $\Delta V_{\mathrm{FB}}(\mathrm{mV})=-1.84+20.5 \Delta \varepsilon_{\mathrm{r}}$, and are consistent with Lin's prediction. However, it is not clear how the temperature dependence of the $V_{\mathrm{FB}}$ shift (Fig. 5 inset) can be explained by Lin's model. At higher temperature, the diffusion of Gd, which was confirmed by the EELS spectra, should actually reduce $\Delta \varepsilon_{\mathrm{r}}$, and the $V_{\mathrm{FB}}$ shifts should therefore become smaller, not larger, as experimentally observed.

In conclusion, we have demonstrated that the $V_{\mathrm{FB}}$ shift in $\mathrm{Gd}_{2} \mathrm{O}_{3}$ capped gate stacks depends on the composition of the underlying gate dielectric and the thermal budget of the process. The $V_{\mathrm{FB}}$ shift appears to be related to the diffusion of the $\mathrm{Gd}$ toward the interface, although the $\mathrm{Gd}_{2} \mathrm{O}_{3}$ layer is not consumed. We believe that the interfacial dipole model can explain these results, but it must be modified to explain that the extent of the $V_{\mathrm{FB}}$ shift is not only controlled by the theoretical dipole moment at the interface, but also by the diffusion rate of the lanthanide element.

${ }^{1}$ H. N. Alshareef, M. A. Quevedo, H. C. Wen, R. Harris, P. Kirsch, P. Majhi, B. H. Lee, R. Jammy, D. J. Lichtenwalner, J. S. Jur, and A. I. Kingon, Appl. Phys. Lett. 89, 232103 (2006).

${ }^{2}$ M. Hong, J. Kwo, A. R. Kortan, J. P. Mannaerts, and A. M. Sergent, Science 283, 1897 (1999).

${ }^{3}$ M. Passlack, N. Medendorp, R. Gregory, and D. Braddock, Appl. Phys. Lett. 83, 5262 (2003).

${ }^{4}$ J. R. Hauser, CVS@2000, NCSU Software, Version 5.0, Department of Electrical and Computer Engineering, NC State University, Raleigh, NC.

${ }^{5}$ D. J. Lichtenwalner, J. S. Jur, A. I. Kingon, M. P. Agustin, Y. Yang, S. Stemmer, L. V. Goncharova, T. Gustafsson, and E. Garfunkel, J. Appl. Phys. 98, 024314 (2005)

${ }^{6}$ M. Copel, E. Cartier, V. Narayanan, M. C. Reuter, S. Guha, and N. Bojarczuk, Appl. Phys. Lett. 81, 4227 (2002).

${ }^{7}$ S. Stemmer, J.-P. Maria, and A. I. Kingon, Appl. Phys. Lett. 79, 102 (2001)

${ }^{8}$ M. Copel, S. Guha, N. Bojarczuk, E. Cartier, V. Narayanan, and V. Paruchuri, Appl. Phys. Lett. 95, 212903 (2009).

${ }^{9}$ Lange's Handbook of Chemistry, 15th ed., edited by J. A. Dean (McGrawHill, New York, 1999), p. 81.

${ }^{10}$ S. Guha, V. K. Paruchuri, M. Copel, V. Narayanan, Y. Y. Wang, P. E. Batson, N. A. Bojarczuk, B. Linder, and B. Doris, Appl. Phys. Lett. 90, 092902 (2007).

${ }^{11}$ P. D. Kirsch, P. Sivasubramani, J. Huang, C. D. Young, M. A. QuevedoLopez, H. C. Wen, H. Alshareef, K. Choi, C. S. Park, K. Freeman, M. M Hussain, G. Bersuker, H. R. Harris, P. Majhi, R. Choi, P. Lysaght, B. H. Lee, H.-H. Tseng, R. Jammy, B. Boscke, D. J. Lichtenwalner, J. S. Jur, and A. I. Kingon, Appl. Phys. Lett. 92, 092901 (2008).

${ }^{12}$ K. Kakushima, K. Okamoto, M. Adachi, K. Tachi, P. Ahmet, K. Tsutsui, N. Sugii, T. Hattori, and H. Iwai, Solid-State Electron. 52, 1280 (2008).

${ }^{13}$ L. Lin and J. Robertson, Microelectron. Eng. 86, 1743 (2009).

${ }^{14}$ H. J. Li and M. I. Gardner, IEEE Electron Device Lett. 26, 441 (2005).

${ }^{15}$ B. P. Linder, V. Narayanan, V. K. Paruchuri, E. Cartier, and S. Kanakasabapathy, Technical Digest SSDM, Tsukuba, 2007, p. 16 